\title{
Treatment of venous thoracic inlet syndrome - Specialties in athletes
}

\section{Behandlung von Schultergürtelvenenthrombosen - Besonderheiten bei Leistungssportlern}

Authors

Nadine Weiske, H. Baumbach, T. Bürger

Affiliation

Klinik für Gefäßchirurgie, Agaplesion-Diakonie-Kliniken

Kassel, Kassel

Key words

Paget-Schroetter syndrome, thrombosis par effort, thoracic inlet syndrome, vascular thoracic outlet syndrome

Schlüsselwörter

Paget-von-Schroetter-Syndrom, Par-effort-Thrombose, Thoracic-inlet-Syndrom, vaskuläres Thoracic-outlet-Syndrom

received 04.05 .2018

accepted 18.11.2018

Bibliography

DOI https://doi.org/10.1055/a-0892-8151

Phlebologie 2020; 49: 10-15

(c) Georg Thieme Verlag KG, Stuttgart · New York

ISSN 0939-978X

Correspondence

Nadine Weiske

Klinik für Gefäßchirurgie

Agaplesion-Diakonie-Kliniken Kassel, Herkulesstraße 34, 34119 Kassel

Tel.: ++49/5 61/10021510

Nadine.weiske@diako-kassel.de

\section{ABSTRACT}

Thoracic inlet syndrome (TIS), also known as Paget-Schroetter syndrome, is a relatively rare form of thoracic outlet syndrome. It is considered to be a consequence of compression and repetitive injury of the subclavian vein between the first ribs and the overlying clavicle, the scalenus anterior muscle, subclavius muscle and costoclavicular ligament. While relatively uncommon, this condition is probably the most frequently encountered vascular disorder in the young, healthy, competitive athlete.
Although the treatment options to be considered for TIS are similar for all patients, competitive athletes often present special circumstances that need to be taken into account in the decision-making process. With early recognition, proper treatment with early thrombolysis, anticoagulation, surgical decompression and rehabilitation, most athletes can return to previous levels of performance within several months. There were 184 patients treated due to compression syndrome of the upper thoracic outlet between 2013-2016. We will report on the therapy and disease management of 5 professional athletes with TIS. In addition to the report, we are presenting the results of a relevant literature search.

\section{ZUSAMMENFASSUNG}

Das Thoracic-inlet-Syndrom (Synonym: Paget-von-SchroetterSyndrom) ist eine seltene Form des Thoracic-outlet-Syndroms. Der venöse Verschluss ist Folge einer wiederholten Kompression im Bereich der oberen Schulterapertur zwischen erster Rippe, Klavikula, Mm. scaleni et subclavius und Lig. costoclaviculare. Obwohl es insgesamt eine seltene Erkrankung ist, zählt sie dennoch bei jungen, gesunden Sportlern zu den häufigsten Gefäßerkrankungen. Die Behandlung eines Thoracic-inlet-Syndroms (TIS) unterscheidet sich grundsätzlich im Jugendalter nicht. Dennoch gilt es bei Athleten oft zusätzliche Umstände zu berücksichtigen. Durch das frühzeitige Stellen der Diagnose kombiniert mit einer raschen Kombinationstherapie (Thrombolyse, Antikoagulation, chirurgische Dekompression und Rehabilitation) können die meisten Sportler ihr vorbestehendes Leistungsniveau nach einigen Monaten wieder erreichen.

Im Zeitraum von 2013-2016 wurden 184 Patienten wegen eines Kompressionssyndroms der oberen Thoraxapertur stationär behandelt. Wir berichten über die Therapie und den Krankheitsverlauf von 5 Profisportlern mit einem TIS. Ergänzt wird der Beitrag durch eine entsprechende Literaturrecherche. 


\section{Introduction}

The possibility of a subclavian vein thrombosis in the context of a thoracic inlet syndrome (TIS) should always be considered in young otherwise healthy patients, who have a sudden onset of arm swelling (especially with activities involving movements above the head, such as lifting weights) or otherwise inexplicable pulmonary emboli [25].

In addition to the swelling, clinical examination reveals a cyanotic discolouration of the skin and an increase in prominent veins over the upper arm, shoulder and chest. The signs and symptoms are often dramatic and associated with pain [10]. There is a striking difference in the findings on the two sides. The condition may be misdiagnosed as an allergic reaction to an insect bite or topical medication. Muscle spasm or tears also have to be ruled out in the individual case.

The diagnosis is usually made with colour-coded duplex ultrasonography. Further cross-sectional modalities (computed tomography (CT) and magnetic resonance imaging (MRI)) may also be used [7]. Venography of the arm is the basis for catheterdirected thrombolysis $>$ Fig. 1a, b) [10].

Once the diagnosis has been confirmed, there are five possible treatment options:

1. Purely conservative with anticoagulation and compression therapy

2. Local thrombolysis with subsequent anticoagulation and compression therapy

3. Local thrombolysis with subsequent surgical decompression of the upper thoracic outlet

4. Surgical decompression of the upper thoracic outlet with subsequent anticoagulation and compression therapy

5. Surgical decompression of the upper thoracic outlet and secondary recanalisation of the venous thrombosis

When considering purely conservative treatment analogous to that given for a deep vein thrombosis of the pelvic or leg veins [8], it must be remembered that these particular patients are all young otherwise healthy people. The risk of a potential lysis-induced complication is therefore extremely low in comparison with thrombolysis performed in other indications. When there is evidence of a compression syndrome, relieving the stress on the externally compromised structures is the only permanent treatment that does not necessitate substantial changes in everyday life. Long-term anticoagulation at any dose is contraindicated or should not be used without at least a critical appraisal in all forms of contact sport and in athletes with a potential risk of injury. Insurance provisions for professional athletes also have to be taken into consideration.

In a competitive athlete, the cause of thrombosis in TIS can mainly be attributed to repeated mechanical compression of the vein between the clavicle and first rib. Besides muscular endurance training and the simultaneous anatomical pressure exerted in the costoclavicular space, a common secondary factor is repetitive chronic strain with the associated risk of injury and development of progressive fibrosis [20]. In competitive athletes, the outbreak of the disease may therefore occur in particular after activities involving abduction, lifting or vigorous movements of

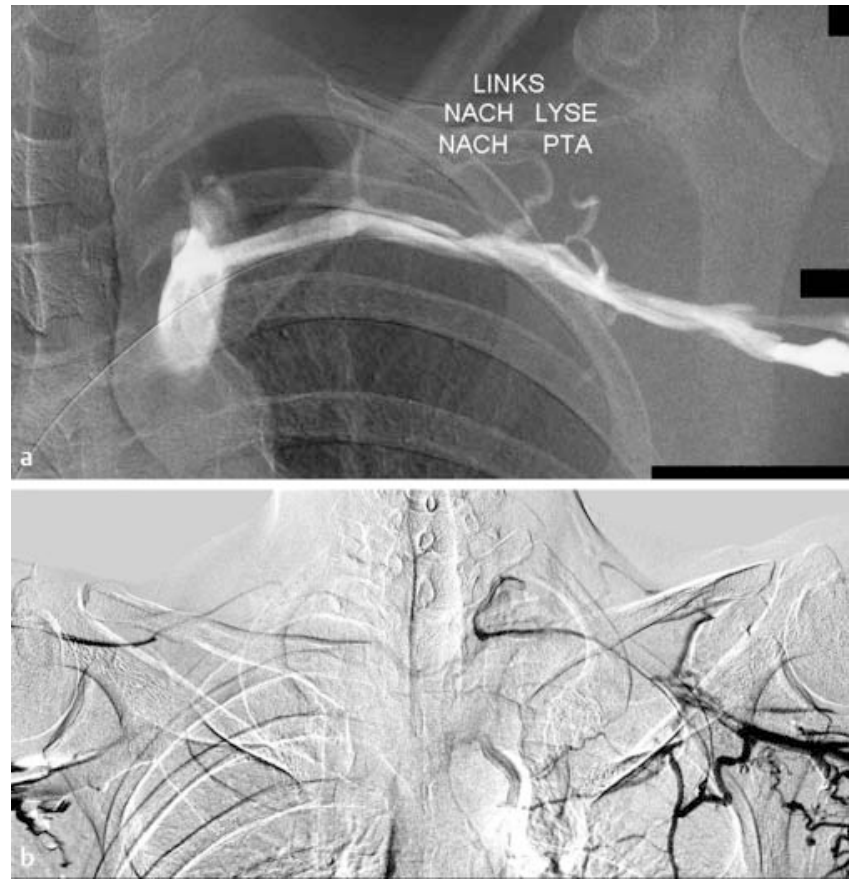

- Fig. 1 a Venography with catheter-directed revascularisation and b evidence of bilateral compression on abduction/elevation in an upright position.

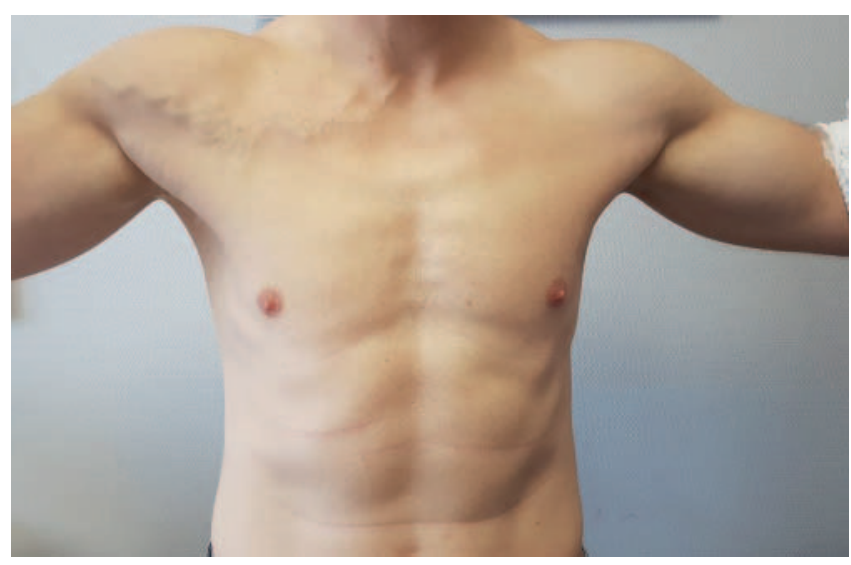

- Fig. 2 Typical clinical picture of a marked collateral circulation in a functional position.

the arm. The average age of onset is between 25 and 35 years of age. It affects men almost twice as frequently as women [10]. Depending on the severity of the condition, the symptoms may be positional or persistent. If the condition becomes chronic, a collateral circulation develops over the chest wall with time ( $\triangleright$ Fig. 2). Pulmonary embolism with dyspnoea and dizziness may also occur in up to $20 \%$ of cases [5].

In a special form of the disease that is seen in some patients, the typical swelling can occur without thrombotic occlusion at various positions of the arm on applying external compression. This particular variant is known as McCleery's syndrome, named after the person who first described it in 1951 [17]. 


\section{Methods}

The present article is based on a presentation given at the $59^{\text {th }}$ Annual Meeting of the German Phlebology Society in Stuttgart (2017).

We analysed the literature on the basis of a PubMed search from January 1980 to 31 May 2018. The search terms (medical subject headings, MeSH) were Paget-Schroetter syndrome, thrombosis par effort, thoracic inlet syndrome and vascular thoracic outlet syndrome. In addition, we included similar articles. The search yielded 2086 hits. The results were analysed for content and further search constraints were subsequently added in an advanced search strategy with the Boolean operators 'and' and 'or'. The literature references that remained were checked for subject value. Further constraints were added with the filters 'randomized control trial' and 'clinical queries'. We took into account only articles published in English or German or with at least a relevant abstract. We compared the data and results obtained with our own experiences.

\section{Results}

We did not find any randomised controlled trials that specifically addressed primary (idiopathic) venous thrombosis of the upper limb. Our search yielded only statements on very heterogeneous causes of upper limb thrombosis due mainly to the insertion of catheter material or in association with cancer. After the further exclusion of publications with a predominantly clinical neurogenic or arterial TOS, only case reports and analyses of small TIS case series remained. The largest-scale publication followed 32 patients over a period of ten years [24]. By further narrowing the search strategy to deep vein thrombosis of the arm in athletes, we found 51 publications. We found publications that specifically cited TIS in athletes in only a further 11 cases $[3,4,6,9,11,14$, $18,19,23,26]$. There were no randomised controlled trials that covered the whole topic.

Out of 276 patients with TOS treated in their own practice (1997-2007), Melby et al. [24] analysed the outcomes in 29 male and 3 female athletes with thrombosis par effort of the upper arm. The right arm was affected in 26 cases (81\%). Seven patients were professional athletes. The interval between the time when the symptoms were first noticed and the diagnosis of venous thrombosis confirmed by venography was $20.2 \pm 5.6$ days (median 4.5 days, range 1-120 days). Preliminary ultrasound examinations were carried out in 21 patients (66\%). However, the findings gave false negatives in six patients (sensitivity $71 \%$ ). Twenty-six patients ( $81 \%$ ) had previously undergone catheter-directed thrombolysis elsewhere. Twelve patients (46\%) from this group had also had balloon angioplasty. One patient had also had a stent inserted. In each case, there was overlapping anticoagulant therapy. A further six patients (19\%) had the primary diagnostic investigation and treatment in-house. All patients were offered secondary surgical decompression of the upper thoracic outlet, irrespective of the time that had already elapsed, although it should ideally be performed within three weeks. Twelve patients had initially received no such recommendation for surgery from the medical facility that first treated them. Due to persistent symptoms (with five cases of recurrent thrombosis despite anticoagulation), these patients were finally transferred and underwent surgery after a considerable delay ( $163.3 \pm 29.2$ days). All patients were operated on by the same surgeon following the same surgical strategy (combination of supraclavicular and infraclavicular access) with full scaleneotomy, plexus neurolysis and removal of the $1^{\text {st }}$ rib. Complete venolysis was carried out in each case. Fourteen patients (44\%) with residual venous stenosis or an operable occlusion opted for a patch plasty or a saphenous vein graft (with a peripheral arteriovenous fistula). The stent that had been inserted previously had fractured. Seven patients needed early revision surgery: three occlusions of the vein graft, two haematomas that required evacuation, one haemothorax in need of drainage and a lymphatic fistula. All 32 patients were able to resume their sporting activities. Even though the outcome was ultimately the same, the inpatient stay was significantly prolonged in patients with delayed and complicated treatment (re-thrombosis).

In the remaining publications, the authors mostly presented a case report on one of their own patients combined with a literature search on the clinical signs, symptoms and treatment of this medical condition. In the more recent literature, we found recommendations for aggressive invasive therapy, with good results [4, $11,19,23,26]$.

In our own patient population in 2013-2016, we treated 184 inpatients with a compression syndrome of the upper thoracic outlet. Surgery was performed on 88 of these patients. Fifty-two patients had a vascular TOS; 12 patients were treated for a purely venous TIS. This specific patient group included five active professional athletes (two surfers, one footballer, one canoeist and one handball player). All of these patients were men ( $\triangleright$ Table 1 ).

We followed the modified treatment schedule given below [5, 13, 22].

After the initial diagnostic work-up, our athletes underwent catheter-directed lysis with urokinase over a maximum of 36 hours. We first gave a bolus dose of 100000 IU followed by $100000 \mathrm{IU} /$ hour via an infusion pump, with a simultaneous administration of unfractionated heparin (usually beginning with $1000 \mathrm{IU} /$ hour). The heparin dose was adjusted according to the monitored coagulation status. In two patients, who initially had undergone only a partial recanalisation with residual stenosis, an additional balloon angioplasty was subsequently performed. Decompression surgery was carried out within a few days to six weeks after the procedure ( $\vee$ Fig. $\mathbf{3}$ ). In addition to the necessary surgical vasolysis and neurolysis, we recommend the complete removal of the cervical/first rib to prevent residual or recurrent problems ( $\nabla$ Fig. 4). All patients were treated with a direct oral anticoagulant (DOAC) and arm immobiliser/ Gilchrist bandage to prevent sudden movements until such time as the definite operation with transaxillary rib resection was performed. Postoperative care was always adapted to the individual situation. Patient-controlled analgesia (PCA) via a pump was used for 2-3 days after the surgical decompression and was then switched to oral analgesia with an individually determined dose of gabapentin, diclofenac and/or Novalgin. Restricted personal training, e. g. on a treadmill, and rehabilitation measures were also possible. In accordance with the literature, anticoagulation was continued for $6-12$ weeks 
- Table 1 Patient characteristics, diagnosis, treatment, and outcome.

\begin{tabular}{|c|c|c|c|c|c|c|c|c|c|c|c|}
\hline Patient & Sex & Age & Sport & Side & $\begin{array}{l}\text { Time until } \\
\text { imaging }\end{array}$ & $\begin{array}{l}\text { Pulmonary } \\
\text { embolism }\end{array}$ & $\begin{array}{l}\text { Throm- } \\
\text { bolysis| } \\
\text { angio- } \\
\text { plasty }\end{array}$ & $\begin{array}{l}\text { Transaxillary } \\
\text { decompres- } \\
\text { sion }\end{array}$ & $\begin{array}{l}\text { Compli- } \\
\text { cations }\end{array}$ & $\begin{array}{l}\text { Duration } \\
\text { of hospi- } \\
\text { tal stay }\end{array}$ & $\begin{array}{l}\text { Competi- } \\
\text { tive sport } \\
\text { resumed }\end{array}$ \\
\hline N.S. & M & 28 & Football & Left & 3.5 months & yes & $+1+$ & + & - & 11 days & 5 months \\
\hline M. H. & M & 28 & Handball & Left & 1 month & no & $+1+$ & + & - & 8 days & 5.5 months \\
\hline J. H. & M & 24 & Surfing & Left & 1 year & yes & \pm & + & - & 26 days & 6 months \\
\hline M.S. & M & 35 & Surfing & Left & 1 week & yes & \pm & + & - & 10 days & 4.5 months \\
\hline J. L. & M & 25 & Canoeing & Right & 2 months & no & \pm & + & - & 12 days & 5.3 months \\
\hline
\end{tabular}

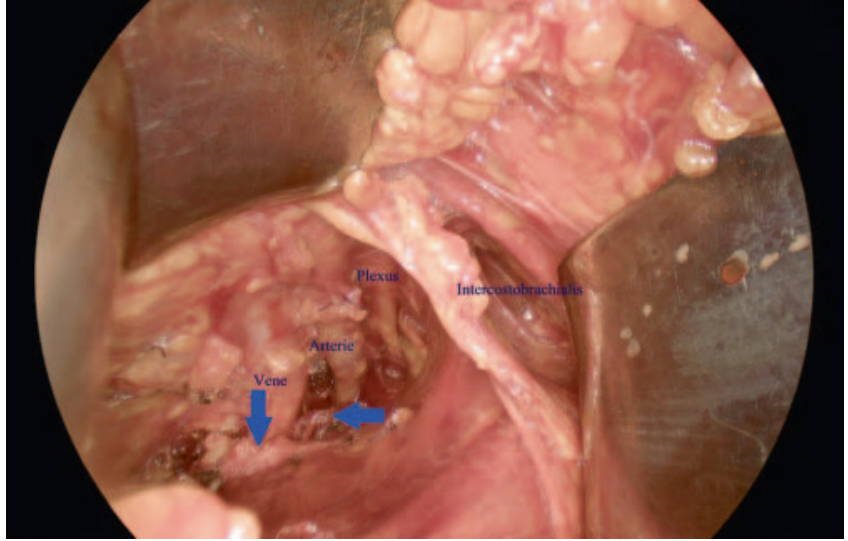

- Fig. 3 Transaxillary access showing the anatomy. The vertical arrow points to the subclavius and the horizontal arrow indicates the anterior scalene muscle that has already been divided.

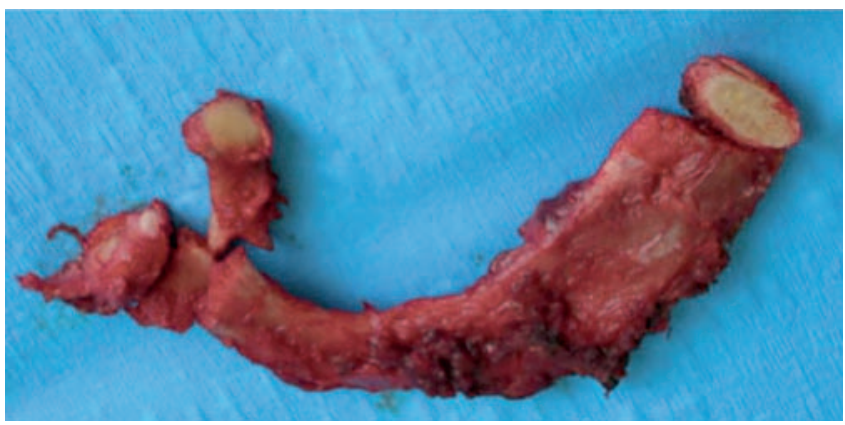

- Fig. 4 Resection of the 1st rib. All anatomical muscle and ligament variants that could potentially compress the vessels are removed, with the exception of the pectoralis minor muscle.

[8]. A return to unrestricted training or team training took 4-6 months ( $\bullet$ Fig. 5). We have not so far had any complications or recurrent thrombosis in our patient population.

A comparison of our results with the data available in the literature is difficult and, in our opinion, would not be justified. The reason for this is that not only the timing, but also the range of treatment for the required comparison was extremely variable. We did not link any conventional open venous reconstruction to this patient population.

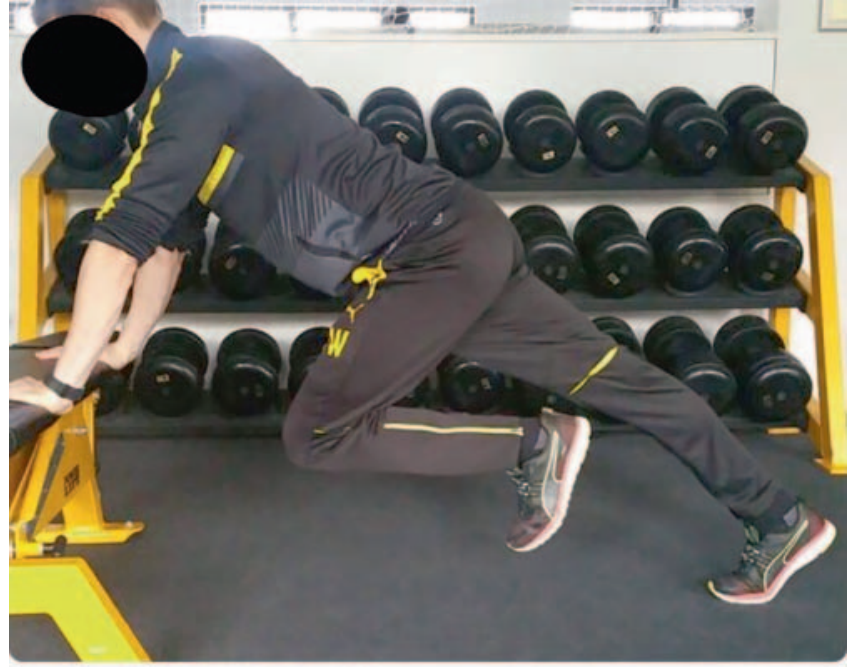

\section{Ist das erlaubt}

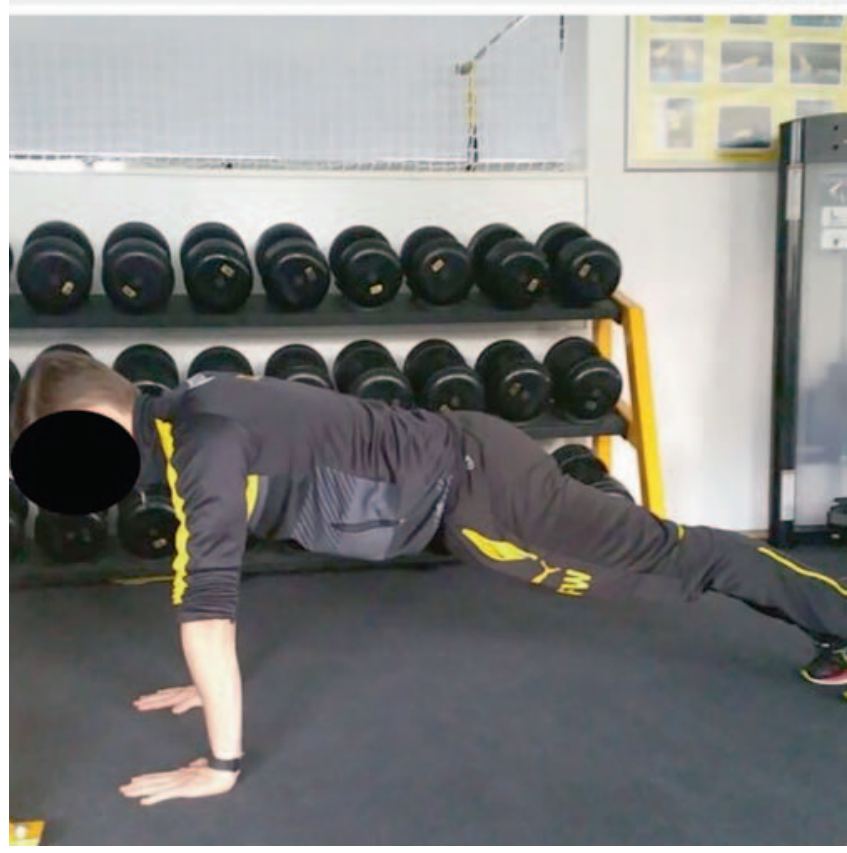

- Fig. 5 Individual follow-up and advice on convalescence, for example, by WhatsApp. 


\section{Discussion}

Deep vein thrombosis in the shoulder girdle has a serious impact on health and sporting activities, especially in competitive athletes. Besides the usual risk factors (Virchow's triad), the congenital narrowing of anatomical structures, such as ligaments, muscle fibres and cervical ribs, generally combined with additional triggers (posture, accident, muscle mass) or taking anabolic steroids must also be considered. The literature also points to the considerable time spent travelling to competitions and repeated localised vascular trauma with particular force and impact.

The treatment goals are to achieve a rapid resolution of symptoms, to lower the risk of pulmonary embolism (some 20-30\%) and to prevent a recurrence of the thrombosis $[1,10,15]$. As this patient group consists of young active athletes, purely conservative measures of anticoagulation and compression are not the treatment of choice in most cases. With this type of treatment, these patients would have to forgo their customary sporting activities because of the increased risk of bleeding (contact sports) and compensate for the long periods when they are out of action making it difficult to resume their active lifestyle. We therefore recommend an active therapeutic approach. For this, a rapid diagnosis is of key importance, which is to be followed by venography and catheter-directed thrombolysis combined with subsequent decompression surgery. Based on the literature and the results in our own patients, the majority of patients, who have successful local lytic therapy and surgical treatment within the first two weeks, have better long-term results [10, 25]. However, the publications do not uniformly assess the precise timing of the operation $[5,10,12]$. Lytic therapy alone without subsequent surgery carries a residual thrombotic risk of $30-70 \%$. Stents should also be avoided. The timing of surgery varied between immediately and an interval of up to three months. Surgical decompression consisted mainly of the transaxillary removal of the first rib with an Atkin's procedure [5], with or without venous reconstruction.

Successful catheter-directed thrombolysis followed by anticoagulation alone and no surgical decompression carries a risk of recurrence as high as $25 \%[16,25]$.

Excellent long-term results have been achieved in specialised centres after combination treatment with thrombolysis and surgical decompression. The five-year patency rate is between $80 \%$ and $90 \%$ [13]. In addition to a rapid diagnosis, prompt referral to an experienced specialist team is essential to achieve the expected good outcome. The longer the occlusion is present, the more complicated the chances of success, even with the necessary expertise.

The experts' consensus-based standard treatment for TIS consists of rapid thrombolysis, anticoagulation and surgical decompression $[2,13,20,21]$. This combination represents the only causal treatment for the condition.

Another major factor in assessing the quality of treatment is the use of a defined follow-up examination and care protocol, such as the one we have established ourselves [10].

In summary, rapid targeted diagnostic investigation and interdisciplinary treatment in an experienced unit makes it possible for these patients to be treated successfully.
Conflict of Interest

The authors declare that they have no conflict of interest.

Literatur

[1] Adams JT, DeWees JA. Effort thrombosis of the axillary and subclavian veins. J Trauma 1971; 11: 923-930

[2] Alla VM, Natarajan N, Kaushik M et al. Paget-Schroetter syndrome: review of pathogenesis and treatment of e ort thrombosis. West J Emerg Med 2010; 11: 358-362

[3] Arko FR, Harris EJ, Zarins CK et al. Vascular complications in high-performance athletes. J Vasc Surg 2001; 33: 935-942

[4] Aquino BC, Barone EJ. "Effort" thrombosis of the axillary and subclavian vein associated with cervical rib and oral contraceptives in a young woman athlete. J Am Board Fam Pract 1989; 2: 208-211

[5] Bürger T, Stegemann E, Baumbach $\mathrm{H}$ et al. V.-subclavia-Thrombose bei Thoracic-inlet-Syndrom. Gefässchirurgie 2016; 8: 558-566

[6] Chaudhry MA, Hajarnavis J. Paget-von Schrotter syndrome: primary subclavian-axillary vein thrombosis in sport activities. Clin J Sport Med 2003; 13: 269-271

[7] Chin EE, Zimmerman PT, Grant EG. Sonographic evaluation of upper extremity deep venous thrombosis. J Ultrasound Med 2005; 24: 829838

[8] Diagnostik und Therapie der Venenthrombose und der Lungenembolie. AWMF Leitlinienregister 2015; Nr. 065-002: 1-94

[9] DiFelice GS, Paletta GA Jr, Phillips BB et al. Effort thrombosis in the elite throwing athlete. Am J Sports Med 2002; 30 (5): 708-712

[10] Hangge P, Rotellini-Colvet L, Deipolyi AR et al. Paget-Schroetter syndrome: treatment of venous thrombosis and outcomes. Cardiovasc Diagn Ther 2017; 7 (Suppl. 3): 285-290

[11] Hurley WL, Comins SA, Green RM et al. Atraumatic subclavian vein thrombosis in a collegiate baseball player: a case report. J Athl Train 2006; 41: 198-200

[12] Illig KA, Donahue D, Duncan A et al. Reporting standards of the society for vascular surgery for thoracic outlet syndrome: Executive summary. J Vasc Surg 2016; 64: 797-802

[13] Illig KA, Doyle AJ. A comprehensive review of Paget-Schroetter syndrome. J Vasc Surg 2010; 51: 1538-1547

[14] Jackson SS, O’Brien MJ. Case report: upper extremity deep venous thrombosis in a 19-year-old baseball player. Phys Sportsmed 2014 42 (2): 163-167

[15] Kucher N. Clinical practice. Deep-vein thrombosis of the upper extremities. N Engl J Med 2011; 364 (9): 861-869

[16] Lee JT, Karwowski JK, Harr E] et al. Long-term thrombotic recurrence after nonoperative management of Paget-Schroetter syndrome. J Vasc Surg 2006; 43: 1236-1243

[17] McCleery RS, Kesterson JE, Kirtley JA et al. Subclavius and anterior scalene muscle compression as a Cause of intermittent obstruction of the subclavian vein. Ann Surg 1951; 133: 588-602

[18] Medler RG, McQueen DA. Effort thrombosis in a young wrestler. A case report. J Bone Joint Surg Am 1993; 75: 1071-1073

[19] Nemmers DW, Thorpe PE, Knibbe MA et al. Upper extremity venous thrombosis. Case report and literature review. Orthop Rev 1990; 19 : 164-172

[20] Nichols AW. Diagnosis and management of thoracic outlet syndrome. Curr Sports Med Rep 2009; 8: 240-249

[21] Nichols AW. The thoracic outlet syndrome in athletes. J Am Board Fam Pract 1996; 9 (5): 346-355 
[22] Ozcinar E, Yaman ND, Cakici M et al. Pharmacomechanical thrombectomy of upper extremity deep vein thrombosis. Int Angiol 2017; 36 (3): 275-280

[23] Skerker RS, Flandry FC. Case presentation: painless arm swelling in a high school football player. Med Sci Sports Exerc 1992; 24: 1185-1189

[24] Spencer JM, Suresh V, Vamsidhar RN et al. Comprehensive surgical management of the competitive athlete with effort thrombosis of the subclavian vein (Paget-Schroetter syndrome). J Vasc Surg 2008; 47: 809-821
[25] Yunce M, Sharma A, Braunstein E et al. A case report on 2 unique presentations of upper extremity deep vein thrombosis. Medicin 2018; 97 (11): e9944

[26] Zell L, Kindermann W, Marschall F et al. Paget-Schroetter syndrome in sports activities-case study and literature review. Angiology 2001; 52: 337-342 